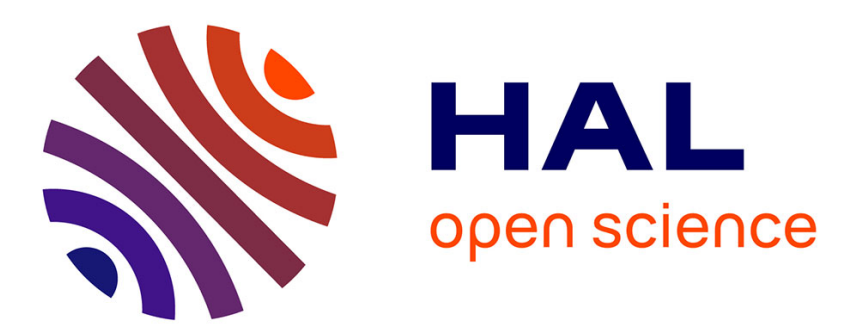

\title{
A novel approach for in-situ detection of machining defects
}

\author{
Yann Quinsat, Lorène Dubreuil, Claire Lartigue
}

\section{To cite this version:}

Yann Quinsat, Lorène Dubreuil, Claire Lartigue. A novel approach for in-situ detection of machining defects. International Journal of Advanced Manufacturing Technology, 2016, 10.1007/s00170-0169478-3 . hal-01374754

\section{HAL Id: hal-01374754 https://hal.science/hal-01374754}

Submitted on 1 Oct 2016

HAL is a multi-disciplinary open access archive for the deposit and dissemination of scientific research documents, whether they are published or not. The documents may come from teaching and research institutions in France or abroad, or from public or private research centers.
L'archive ouverte pluridisciplinaire HAL, est destinée au dépôt et à la diffusion de documents scientifiques de niveau recherche, publiés ou non, émanant des établissements d'enseignement et de recherche français ou étrangers, des laboratoires publics ou privés. 


\title{
A novel approach for in-situ detection of machining defects
}

\author{
Yann Quinsat • Lorène Dubreuil · Claire lartigue
}

Received: date / Accepted: date

\begin{abstract}
Integrating inspection procedures in the machining process contributes to process optimization. The use of $i n$-situ measurement allows a better reactivity for corrective actions. However, to be highly efficient, Machining and Inspection Process Planning must reach a high level of integration. It is here essential to focus on the compromise measurement time vs precision: the time dedicated to inspection must be limited, but not to the detriment of measurement quality. A measurement process for in-situ machining defect detection is proposed based on a stereo-DIC. The proposed method allows the direct comparison between images of the machined part and the CAD model by means of a CADmodel based calibration method which links the camera frame to the CAM frame. Therefore, starting from a meshed-model representation of the part, local regions of interest are defined corresponding to the projection of each facet onto the two images. An optimization of each facet configuration is then performed in order to minimize a cross-correlation coefficient, and the obtained facet displacement is used to detect machining defects. The robustness of the method is assessed through an illustration of measurement in the machine-tool environment.
\end{abstract}

Keywords In-situ Measurement · Calibration . Stereocorrelation · Inspection Process Planning

Y. Quinsat

LURPA, ENS Cachan, Université Paris Sud, Université ParisSaclay

61 avenue du président Wilson, F-94235 Cachan, France

Tel.: +33147402213

Fax: +33147402220

E-mail: yann.quinsat@ens-cachan.fr

\section{Introduction}

Machining process monitoring is an essential challenge for the control of production costs and of the quality of manufactured parts. The process-setting stage generally requires $100 \%$ inspection of the first manufactured parts. Inspection procedures are increasingly integrated in the machining process. Associated benefits include high speed of inspection, measurement flexibility, on-machine inspection, and the possibility of $100 \%$ inspection [3]. In the context of integrated inspection, three types of measurement can be differentiated: in-situ measurement, in-process measurement, and onmachine measurement [18].

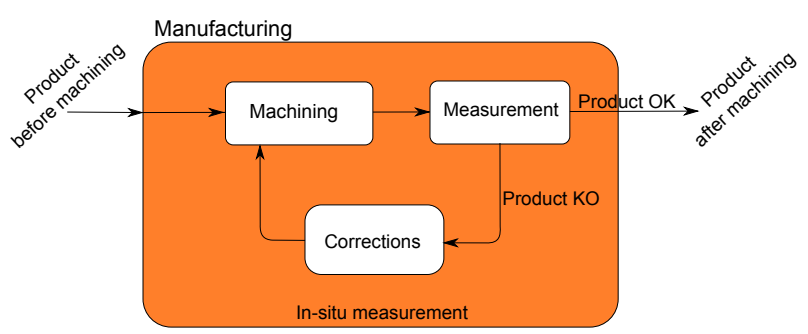

Fig. 1 Context of in-situ measurement [7]

In-situ measurement encompasses all the measurement techniques which are performed in the machine environment, more particularly in-process measurement, and on-machine measurement. During in-process measurement, the manufacturing process is not stopped, and inspection is carried out simultaneously with part machining. In this case, the measurement is not necessarily the geometry of the part but can be another characteristic such as cutting forces, spindle vibrations, and so on. On the other hand, on-machine measurement is 
performed while the part is still located in the machinetool but while the machining process is stopped. Here, the machine-tool holds the sensor, classically a touch probe, and allows sensor displacements as on classical Coordinate Measuring Machines (CMM) [12]. For insitu measurement of geometry, non-contact techniques are more and more used, as they represent a good compromise between rapidity and resolution. For instance, they are used to control roughness defects during turning [16], to measure geometrical deviations during 3axis milling [14] or to monitor 3D surface topography during polishing[15].

Whatever the sensor used, in-situ measurement enables rapid decision making regarding the part's geometrical conformity, as the measurement stage is performed during the manufacturing phase. A direct link exists between Computed-Aided Inspection (CAI) and Computer-Aided Manufacturing (CAM), which can lead to a complete integrated manufacturing process [3]. However, a high level of integration requires the process planning to be changed in order to adapt to the specificity of in-situ measurements. In their approach, Cho and Seo. [5] present an inspection process planning strategy for on-machine measurement of sculptured surfaces using a touch probe. They base their approach on the concept of $\mathrm{CAD} / \mathrm{CAM} / \mathrm{CAI}$ integration, meaning that $\mathrm{CAD} / \mathrm{CAM}$ data are integrated into the CAI process. In particular, they propose to use cutter contact points to define measuring-point locations in order to avoid inspection errors induced by cusp heights. Lee et al. [11] and Cho et al. [4] base their approach on features and on two main steps: a global step and a local step. The global step gathers and orders all the features prior to the measurement step, during which the probe trajectories are defined for each feature. More recently, Zhao $[19,20]$ developed a data model foron-machine inspection using a touch probe using STEP-NC format. The main drawback associated with in-situ measurement is that the machine is unavailable during the measurement phase. This can lead to a loss of productivity. As a result, Machining and Inspection Process Planning (MIPP) turns out to be the key point to reach a high level of integration. It thus becomes essential to focus on the compromise measurement time vs precision. Therefore, we propose to decompose the measurement operation into two steps. The first step, referred to as pre-measurement, aims at determining and locating potential defects without necessarily quantifying them. If defects are detected during the pre-measurement stage, then the measurement operation is started to quantify the defects before correcting the machining process. As the pre-measurement acts as a decision-support tool, a vision-based system is adopted because it can easily be implemented within the machine-tool environment and can acquired data swiftly.

This paper is organized as follows. The next section presents our approach for Machining and Inspection Process Planning (MIPP) based on in-situ measurements. A SySml formalism is adopted to clearly bring out resources, data and data exchanges. Integration of inspection within the manufacturing process leads to new challenges, such as adapting manufacturing and inspection plans so as not to penalize production time and nor, consequently, total costs. The originality of the process planning we propose is to include a step of pre-measurement to localize defects before the measurement step, which can thus be initiated if needed. Section 3 is dedicated to the description of this premeasurement step, which is performed based on vision, and uses stereo-DIC (Digital Image Correlation). The novel method we develop takes advantage of the CADmodel based calibration method that makes the manufacturing and measurement frames coincide. A point that may also be emphasized is the use of a tessellated representation of the $\mathrm{CAD}$ model to define wise regions of interest for stereo-DIC. Finally, in section 4 we assess the method, through a test case performed in real machine-tool environment.

\section{Machining and Inspection process planning}

Process planning translates information into the process plan which specifies how the product is manufactured. This includes the definition of operations, operation sequences, set-up instructions, and step-by-step work instructions. In order to make more effective use of in-situ measurement, we propose to model all process resources, including resources linked to inspection process.

\subsection{Data description}

Systems, resources and data exchanges are modeled using the Systems Modeling Language (SysML), as it is well-adapted for system engineering applications. The system we consider is the whole manufacturing process including machining and in-situ measurement operations. In SysML, a block can be a system, a device, software or data. The block diagramm describes the main relationships between blocks. Therefore, the differences between the machining process with off-line measurement and the one with in-situ measurement can be illustrated through block diagramms as it is displayed in figure 2 . 


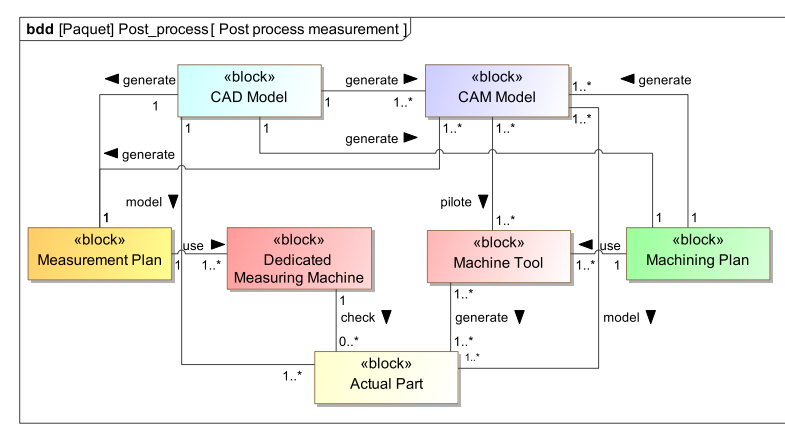

(a) SysML block definition diagram for off-line measurement

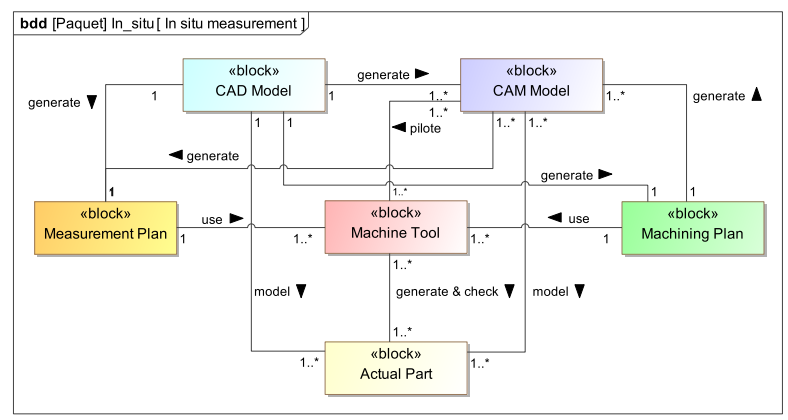

(b) SysML block definition diagram for in-situ measurement

Fig. 2 Description of the different inspection processes

Classically, when measurement operations are performed off-line, seven blocks (figure 2(a)) represent the process as proposed in the list below:

- CAD model,

- CAM model,

- Machining plan,

- Measurement plan,

- Actual part,

- Machine-tool,

- Dedicated Measuring Machine.

The CAD model results from the expression of the design intents into the part geometry, generally as a set of features, along with dimensional and geometrical specifications associated with functional surfaces (GD\&T). The CAD model is the starting point of the process.

The CAM model enables the representation of the different part states throughout the whole process. It includes the machining features, which correspond to geometrical features and to associated machining processes. Intermediate states can be represented by inprocess models that generally consist of a polyhedral mesh, resulting from NC-simulation, definition of the resources (tool, fixtures, and machine-tools), and manufacturing tolerances.

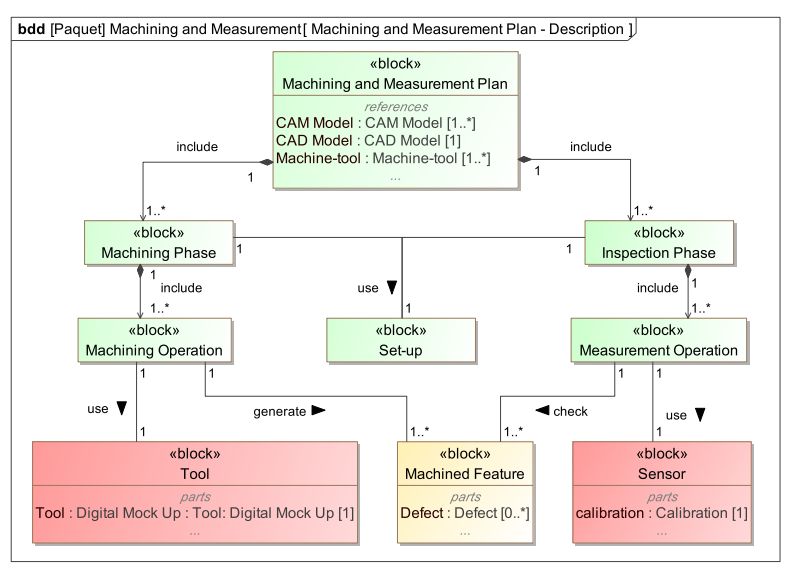

Fig. 3 Modeling of the Machining/Inspection process plan

The machining plan provides ordered operation sequences, step-by-step work instructions, including dimensions related to each individual operation, machining parameters, and set-up instructions. The measurement plan plays the same role as the machining plan for measuring operations. The actual part consists of the machined features that can be related to the machining features of the CAM model, and to the features of the CAD model. Each machined feature presents various geometrical deviations that must be measured. The machine-tool, defined as a poly-articulated structure, allows part manufacturing. Tools, holders, and set-ups are generally associated to the machine-tool.

The dedicated measuring machine is the same element as the machine-tool, but for measuring operations with sensors instead of tools. In-situ measurement reduces the number of blocks from 7 to 5 by removing the dedicated measuring machine and by merging machining and measuring plans (figure 2(b)). Data exchanges are thus facilitated, and machining and measuring operations can be performed during the same phase using the same part set-up and, as a result, in the same reference frame, which is an interesting advantage, as we will see later. With the sysML model, it becomes possible to identify the components, their sub-components and how data flow between them. Such a modeling also brings out relevant parameters for an effective and efficient manufacturing process. Two key points are thus highlighted to enhance in-situ measurement efficiency. First, the measurement plan does not only rely on the CAD model, but also depends on the CAM model, and more precisely on the in-process model (obtained at the end of each machining operation). Secondly, and as a consequence, measurement results have to be directly linked to the CAM model. 


\subsection{Activity description}

Considering the previous modeling, the sequencing of machining and measurement operations can be represented through an activity diagram as displayed in figure 4. Our approach brings out three activities in the machine-tool. The first operation, as usual, is the machining operation. It is followed by in-situ measurement, which verifies the conformity at the end of the machining operation. The last activity corresponds to possible corrective operations in case of non conformity.

The measurement step requires the selection of a sensor according to the characteristic to be evaluated. The issue of sensor selection has been addressed in previous studies $[6,13]$ with the aim of finding the most appropriate sensor depending on capacity and quality criteria (resolution, acquisition time, trueness, noise, etc) well-adapted to the defect to be measured. One major difficulty in making the best choice is to predict the defect location and its scale (micro or macrodefect). On the other hand, as in-situ measurement makes the machine-tool unavailable for machining during measurement phases, measurement time can become highly penalizing on productivity. Therefore, we propose to add an operation specifically dedicated to defect detection before the measurement (figure 4). This operation, called pre-measurement, aims at determining and locating potential areas of deviations without necessarily quantifying them. To be efficient, this premeasurement step can be performed using a visionbased system, acting as a decision-support tool. The measurement operation is only started if the system detects deviations on the part due to the machining operation. The choice of a stereo-vision system is motivated by its simplicity of implementation within the machinetool environment, and its rapidity to acquire data (or images). The method we propose for image processing relies on stereo-digital image correlation (StereoDIC). Its originality lies in the use of the CAD model, or the in-process model, to support image correlation. Furthermore, the simple analysis of correlation scores enables the detection and the location of machining defects.

\section{Stereo-vision system for in-situ detection of part defects}

The stereo-vision system is an analysis tool for deciding whether the measurement operation is necessary or not. The system we propose, which consists of 2 cameras, is located in the machine-tool environment and allows simple on-machine measurements (figure 5). Classically, stereo-vision system gives the possibility of re- constructing a 3D part from a pair of corresponding images. 3D point coordinates are calculated from their relative position in two image pairs thanks to the calibration. Once two images of the part are acquired, it is necessary to match corresponding points on the two images. For this purpose, Stereo-DIC is used in our study. As previously said, measurement operations must be highly efficient so as not to alter process productivity. To answer this issue, the proposed method takes advantages of the CAD model for both system calibration and image correlation.

\subsection{Calibration principle [7]}

Calibration aims at identifying the model parameters that define the relationship between the coordinates of a $2 \mathrm{D}$ point, expressed in the picture frame $R_{p}$, and the coordinates of a $3 \mathrm{D}$ point expressed in the reference frame $R_{w}$.

$$
\left(\begin{array}{c}
u \\
v \\
1
\end{array}\right)_{R_{p}}=\text { A.D.P.T. }\left(\begin{array}{c}
X \\
Y \\
Z \\
1
\end{array}\right)_{R_{w}}=M \cdot T \cdot\left(\begin{array}{c}
X \\
Y \\
Z \\
1
\end{array}\right)_{R_{w}}
$$

Based on the pinhole model (Fig. 6), the relationship between $R_{p}$ and $R_{w}$ results from the combination of the geometric transformations $A, D, P$, and $T$ as defined in (Eq. 1). The matrix $A$ corresponds to the affine transformation from the "center" picture frame $R_{r}$ to the picture frame $R_{p}$. The transformation $P$ is a perspective projection of $R_{c}$, the frame attached to the camera, into the retinal plane $R_{r}$. The transformation $D$ corresponds to camera distortions. The parameters associated with the matrices $A, D$, and $P$ correspond to the internal calibration parameters also called the intrinsic parameters: the focal $f_{c}$, the picture center position $C_{c u}$ and $C_{c v}$, the pixel size $s_{p}$, the pixel number $n_{p}$, and the distortion parameters $k_{i}$ (for $\mathrm{i}=1$ to 5 ).

The transformation $T$ between the reference frame $R_{w}$ and the camera frame $R_{c}$ corresponds to the combination of a rotation $R(3 * 3)$ and of a translation $t$. $T$, and can be described thanks to a homogeneous matrix. Its parameters are called extrinsic parameters.

To be efficient, in-situ vision must be performed in the same reference frame than that of the machining operation. This implies that the measurement frame and the CAM frame coincide, and as a result, the calibration of the vision system must be carried out in the CAM frame. For this purpose, in a previous study, we proposed a CAD model-based approach for calibrating a vision-system [7]. In this approach, the reference frame is the CAM frame $R_{w}=R_{C A M}$. During in-situ vision, 


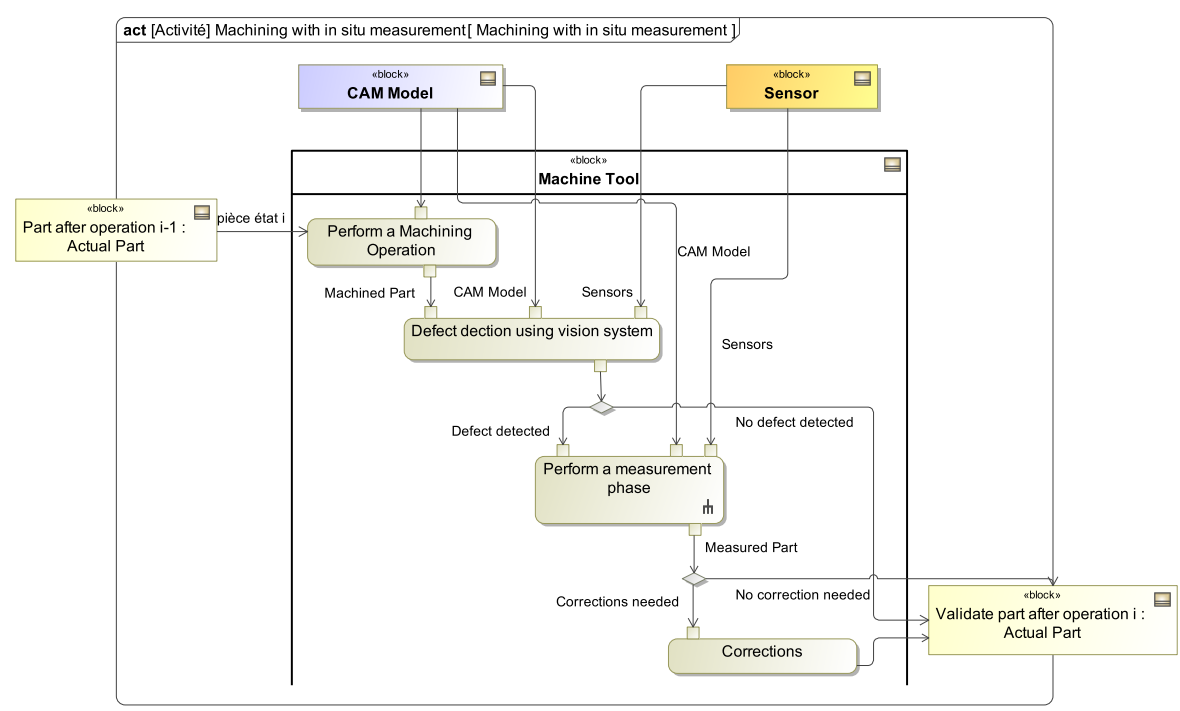

Fig. 4 SysML activity diagram

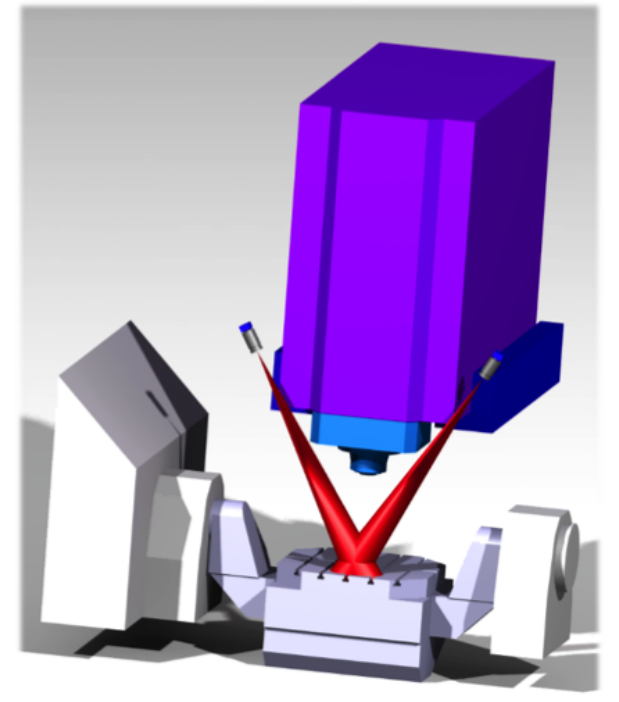

Fig. 5 Description of the vision-based system

intrinsic parameters are fixed and do not vary. Therefore, the transformation $M=A . D . P$ between $R_{p}$ and $R_{c}$ is completely defined, and the objective is to identify the extrinsic parameters i.e. the elements of the transformation matrix $T$. The method proposed in [7] uses a correspondence between a set of $3 \mathrm{D}$ features of the setup CAD model and the corresponding $2 \mathrm{D}$ features in the image of the actual machining set-up to determine the matrix $T$ using the least-square method. Once the correspondence is established, the transformation $G$ between $R_{p}$ and $R_{C A M}$ is known, and $T$ is simply given by:

$T=M^{-1} \cdot G$
The calibration so defined directly links the camera frame $R_{c}$ to the CAM frame $R_{C A M}$. A way to assess the quality of calibration is to evaluate the noise of calibration [10]. This evaluation relies on the calculation of the residual vector $V$ :

$V=\left(I-J \cdot\left(J^{T} \cdot J\right)^{-1} \cdot J\right) \cdot E(\Phi)$

Where $\Phi$ is the vector of the intrinsic parameters to be identified, $E(\Phi)$ is the error vector, and $J=\left[\frac{\partial E}{\partial \Phi}\right]_{\Phi}$. It is thus possible to calculate an estimation of the calibration noise according to the system redundancy $r$, i.e the difference between the number of independent equations and the number of unknowns:

$\sigma_{0}^{2}=\frac{V^{T} \cdot V}{r}$
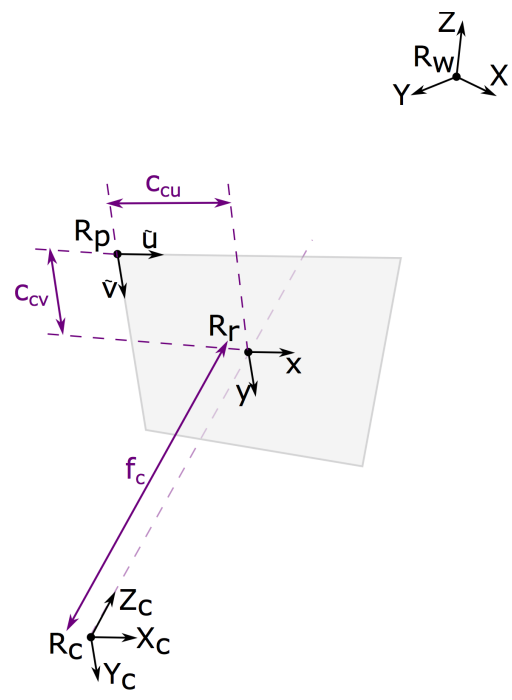

Fig. 6 Pinhole model 


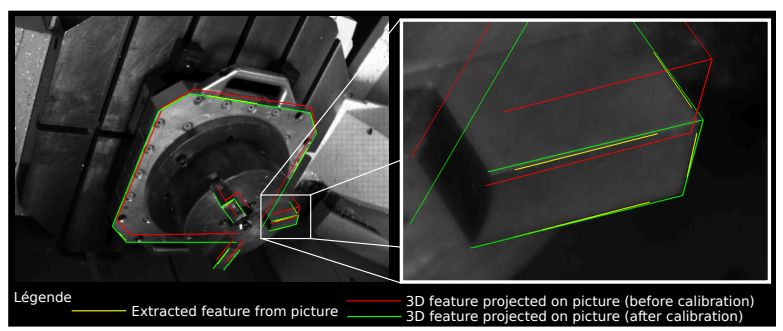

Fig. 7 Calibration from set-up measurement in real environment from [7]

It is worth noting that the calibration noise is defined for each camera, which means a noise, expressed in pixels, is thus associated with both images, the right and the left. For instance, in our approach, calibration is performed using the set-up represented in figure 7 . The estimation of the calibration noise using equation 4 provides respectively $\sigma_{0}^{l}=1.64$ pixel for the left camera and $\sigma_{0}^{r}=1.68$ pixel for the right camera. As it will be highlighted, the defect detection method detailed in the next section is very dependent on this parameter, reflecting calibration quality.

\subsection{Stereo-DIC for defect detection}

The method we propose to detect and localize part defects relies on the stereo-Digital Image Correlation principle (Stereo-DIC). The aim is to determine areas of the part being machined that do not correspond to the CAD model (or in-process model) using stereo-DIC. The originality or our approach lies in the use of the CAD model to define the cross-correlation coefficient between the two images.

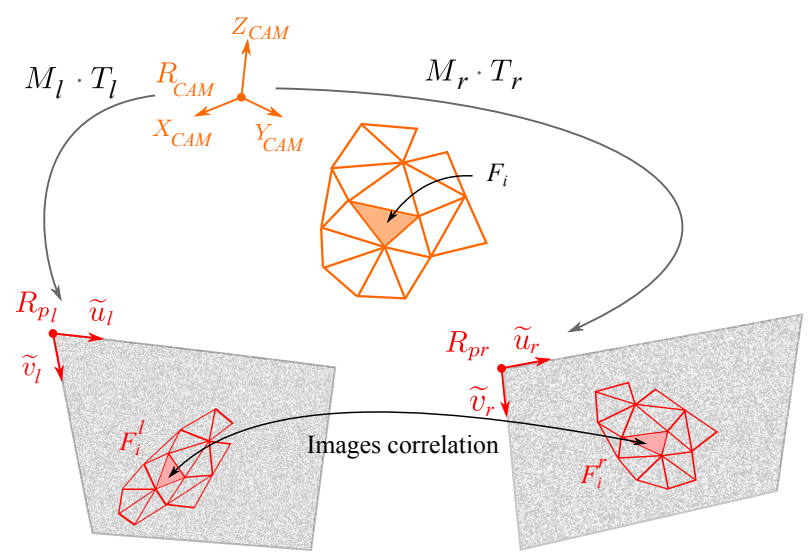

Fig. 8 Definition of the Region Of Interest (ROI) using the CAD model

\subsubsection{Calculation of the cross-correlation coefficient}

Each camera of the stereo-vision system gives a 2D image of the part surface. Prior to 3D surface reconstruction, it is necessary to define correspondence between the two images. Stereo-DIC methods provide some solutions to this issue, also called image matching problem. As it is not possible to find the correspondence of a single pixel in one image in a second image, the idea is to find the correspondence of a small neighborhood around the pixel of interest, referred to as Region Of Interest (ROI). DIC algorithms compare the gray-levels of ROI in the two images using a cross-correlation coefficient that accounts for the similarity of the 2 ROI. Commonly, the ROI is rectangular and identical for the 2 images. These methods are largely used for measuring surface displacements under mechanical loads. In these cases, the shape of the ROI may differ from one image to the other, in order to take the possible deformation of the ROI during measurement into account [17]. As far as stereo-DIC is concerned, it is worth noting that the part surface projection onto the two image-planes does not necessarily have the same geometry. The shape of each ROI must thus be adapted to the projected surface portion so that the calculation of the correlation coefficient can be consistent $[8,9]$. The originality of the proposed method is the use of the CAD-model to define the shape of each ROI. More particularly, the CADmodel is meshed through a STL format. Considering that calibration is carried out (see section 3.1), each triangular facet of the mesh can be projected onto the image-plane of each camera (figure 9). The projection provides triangles of different sizes and orientations depending on the location and orientation of each facet relatively to each optical axis (figures 9 (a) and $9(\mathrm{c})$ ). Nevertheless, the shape of the facet is preserved and can be used as a ROI adapted to the local shape of the part.

Therefore, each 3D facet of the meshed CAD model is considered as a ROI. Projections of the considered ROI can then be compared without additional deformation, as is classical performed, and the cross-correlation coefficient can be easily calculated. In order to improve similarity evaluation, the ROI is sampled to provide a relatively significant number of evaluation points. Let us consider a facet $F_{i}$, and the $n$ evaluation points $X_{i, j}$ (figure $9(\mathrm{~b})$ ); the cross-correlation coefficient for $F_{i}$ is calculated as follows:

$\chi_{i}^{2}=\sum_{j=1}^{n}\left|f^{l}\left(x_{i, j}^{l}\right)-f^{r}\left(x_{i, j}^{r}\right)\right|^{2}$

where $x_{i, j}^{l}$ and $x_{i, j}^{r}$ represent the projection of $X_{i, j}$ on the images, respectively, left and right, and $f^{l}\left(x_{i, j}^{l}\right)$ 


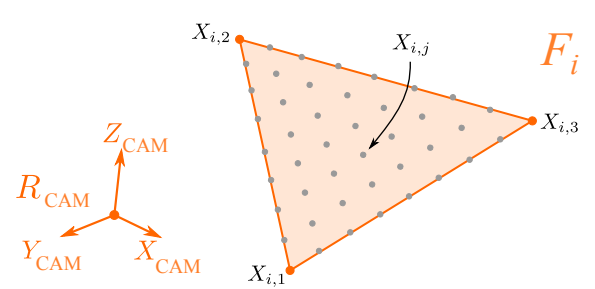

(a) Sampling of the 3D facet
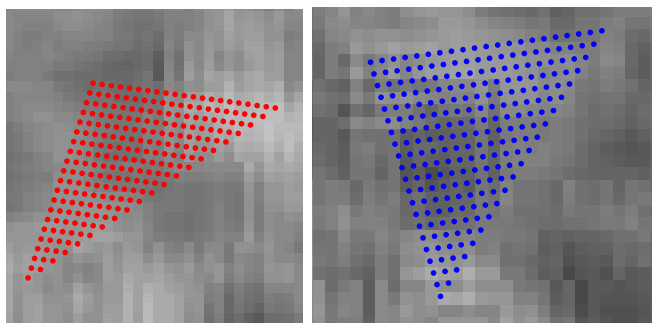

(b) Sampled projec- (c) Sampled projection tion of the left image of the right image

Fig. 9 Calculation of the cross-correlation coefficient from the sampled ROI

and $f^{r}\left(x_{i, j}^{r}\right)$, the associated gray-levels. These values are obtained thanks to a bi-linear interpolation from the projected points. The cross-correlation coefficient $\chi_{i}^{2}$ is similar to a SSD (Sum of Squared Differences) coefficient. It is used here to detect possible deviations between the machined part and its CAD-model. Indeed, if the coefficient $\chi_{i}^{2}$ is low, the measured part locally matches its CAD-model, and the part does not present local deviations. On the opposite, if a local deviation exists, the coefficient $\chi_{i}^{2}$ will be higher. This analysis can be carried out for all the visible areas of the part, and as a result, provides a cartography of the regions presenting deviations relatively to the CAD model.

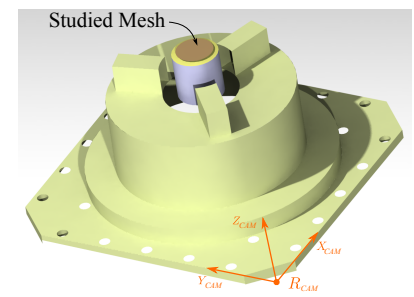

(a) Actual part in machining set-up

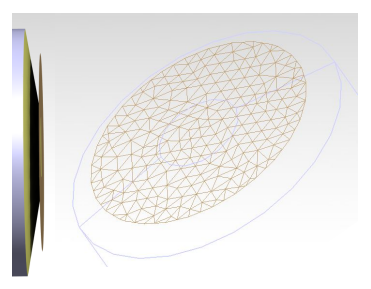

(b) Planar mesh model
Fig. 10 Description of the test part

To illustrate our approach, we propose to measure a test part with a predefined defect. The part is a plane surface, limited by a circle, and it is meshed as proposed in figure $10(\mathrm{~b})$. The part actually machined consists of a truncated cone with a very high apex anglefigure 10(a)), so that the machined part is close to its model but presents a predictable region of defects (figure 12(a)). As a consequence, the correlation coefficient, computed from equation 5 for the left and right images of the part (figure 11), should be low on the middle region corresponding to the planar portion (in blue in figure 12(a)) and, on the opposite, high on the conical region (in red and purple in figure 12(a)). During machining, the part is positioned on the set-up that supports the system calibration. In other words, the CAM frame is attached to the part set-up (figure 10(a)). At the end of machining, the part, still located on its set-up, is measured using the stereo-vision system. Our approach is thus applied for each facet of the model, and the values of the calculated correlation coefficients are reported in figure $12(\mathrm{~b})$.
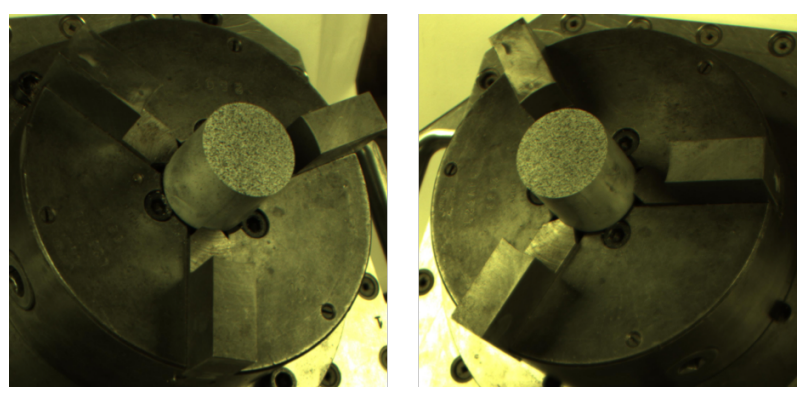

Fig. 11 Left and right pictures of the test part

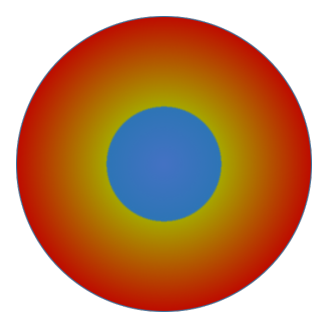

(a) Predictable deviations to be evaluated

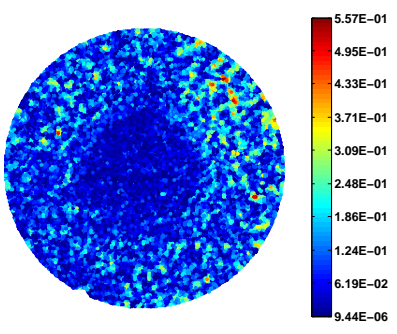

(b) Initial residual
Fig. 12 Predictable and measured residual

As expected, facets of the middle region present a low coefficient. But for the conical region, no significant trend is observable : some facets have a high coefficient and others do not. This can be explained by the combination of two main factors. The first one is obviously the presence of the defect itself. But variations of the correlation coefficient also likely result from calibration errors. Indeed, due to calibration errors, the projection of the facet in each image-plane is not perfectly 
located.Therefore, in each image-plane, the considered ROI is lightly shifted from its ideal position, and this provides some errors in the correlation coefficient calculation. As detailed in the previous section, (see 3.1), calibration errors can be expressed through the calibration noise $\sigma_{0}$ associated with each camera (equation 4 ). Searching regions of high correlation coefficient is then not a sufficient condition to identify regions of defects. It is necessary to optimize the position and orientation of each facet in order to minimize the correlation coefficient. After optimization, regions of deviations will be identified by thresholding the displacements based on the calibration noise coefficient.

\subsubsection{Optimization of facet configuration}

Each facet $F_{i}$ is characterized by the position of its barycentre $X_{B_{i}}$ (in the CAM frame) and its normal vector (defining its orientation) $\mathbf{n}_{\mathbf{i}}$ (figure 13). Let us consider the small displacement of the facet $F_{i}$, denoted $\mathbf{d B}_{\mathbf{i}}$ [2]. This displacement accounts for the rigid body movement of the facet, combination of a translation $\mathbf{T}=\left[\begin{array}{lll}d u_{i} & d v_{i} & d w_{i}\end{array}\right]^{t}$ and three rotations $d \alpha_{i}, d \beta_{i}, d \gamma_{i}$, which are assumed to be small enough to linearize the corresponding rotation matrix at the first order.

$$
\mathbf{d B}_{\mathbf{i}}=\left[\begin{array}{llllll}
d \alpha_{i} & d \beta_{i} & d \gamma_{i} & d u_{i} & d v_{i} & d w_{i}
\end{array}\right]^{t}
$$

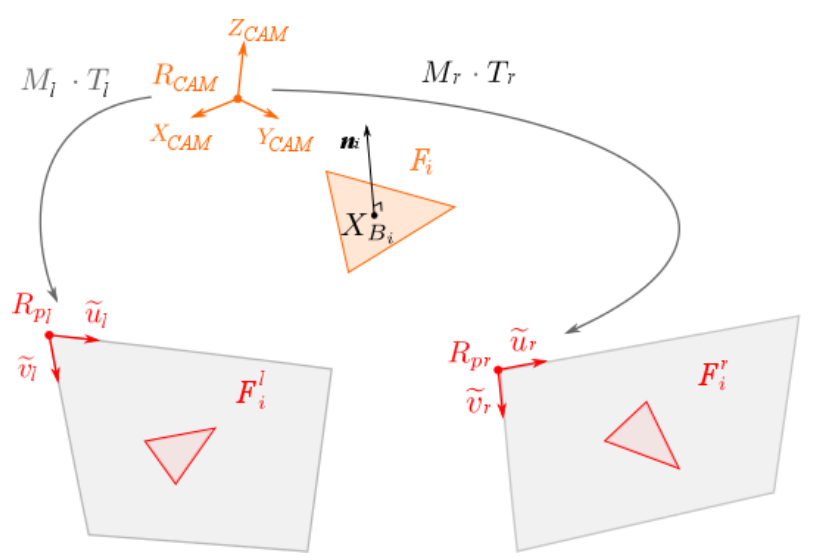

Fig. 13 Optimization of the facet displacement

Let us consider an evaluation point $X_{i, j}$, and $x_{i, j}^{l}$ and $x_{i, j}^{r}$ its projections on the left and right images respectively figures $9(\mathrm{~b})$ ). Associated displacements are denoted $d x_{i, j}^{l}$ and $d x_{i, j}^{r}$. Using equation 5 , the crosscorrelation coefficient can thus be calculated as follows:

$\chi_{i}^{2}=\sum_{j=1}^{n}\left(f^{l}\left(x_{i, j}^{l}+d x_{i, j}^{l}\right)-f^{r}\left(x_{i, j}^{r}+d x_{i, j}^{r}\right)\right)^{2}$
The linearization at the first order of the previous equation leads to the following function to be optimized:

$$
\begin{aligned}
& \chi_{l i n, i}^{2}=\sum_{j=1}^{n}\left(f^{l}\left(x_{i, j}^{l}\right)+\left[\mathbf{G}^{l}\right]\left(x_{i, j}^{l}\right)-f^{r}\left(x_{i, j}^{r}\right)-\left[\mathbf{G}^{r}\right]\left(x_{i, j}^{r}\right)\right)^{2}(8) \\
& \text { with } \\
& G^{l, r}\left(x_{i, j}^{l, r}\right)=\left(\nabla f^{l, r} \cdot d x_{i, j}^{l, r}\right)\left(x_{i, j}^{l, r}\right)
\end{aligned}
$$

and

$d x^{l, r}=\frac{\partial x_{i, j}^{l, r}}{\partial X_{i, j}} \cdot \frac{\partial X_{i, j}}{\partial B_{i}} \cdot \mathbf{d B}_{\mathbf{i}}$

The optimization problem is solved using the NewtonRaphson method for which the small displacements $\mathbf{d B}_{\mathbf{i}}$ are calculated at each iteration. The application of the approach to the previous part clearly highlights a decrease in the correlation coefficient for all the facets (figure 14(b)). Some variations are still present mainly due to an observed heterogeneity in the lighting during the measurement. The method provides a new position and a new orientation of each facet, which minimize the correlation coefficient. This new configuration thus induces a shift of its projection into each image-plane relatively to the initial configuration, and it becomes possible to define the facet displacement, expressed in pixels, in each image-plane, $D_{i}^{l}$ and $D_{i}^{r}$. The displacement is defined here as the maximum of each facet vertex displacement. The study of the displacements in each image will help us detect region of defects, as explained next.

\subsection{Detection of machining defects}

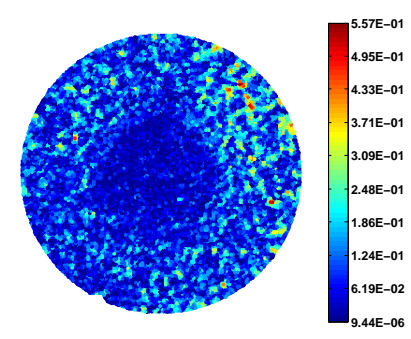

(a) Initial residual

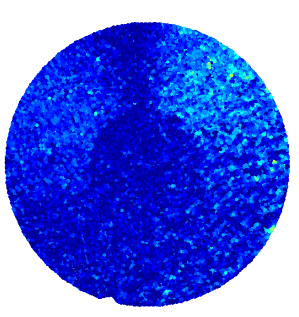

(b) Residual after optimisation
Fig. 14 Maps of the correlation coefficient

Our method to identify regions of defects relies on the calibration noise, which can be assimilated to the measurement resolution. As aforementioned, the calibration noise is denoted $\sigma_{0}^{l}$ for the left camera, and $\sigma_{0}^{d}$ 
for the right camera. If $D_{i}^{l}$ (respectively $D_{i}^{d}$ ) is greater than the calibration noise (respectively $\sigma_{0}^{l}$ and $\sigma_{0}^{r}$ ) then the zone of the part corresponding to the facet presents a defect. On the opposite, if the displacement is lesser than the calibration noise, then the result is in the calibration uncertainty, and it may be assumed that the part does not present a defect. This method is applied to the previous part. Estimated displacements are displayed in figures 15(b) and 15(a). Displacements are thus compared to the calibration noise. Results reported in (figure 15(c)) clearly brings out the region of defects. When this region is compared to the expected results (exterior of the red line corresponding to the conical region of the part), a very good matching is observed. This highlights the relevancy of our method for locating regions of defects using Stereo-DIC. The next section is dedicated to the assessment of the approach in real conditions with the comparison to another measuring system.

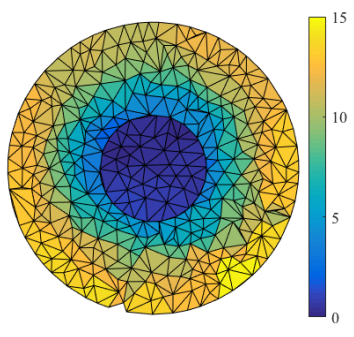

(a) Estimation of the facet displacements $D_{i}^{g}$ (in pixels) in the left camera

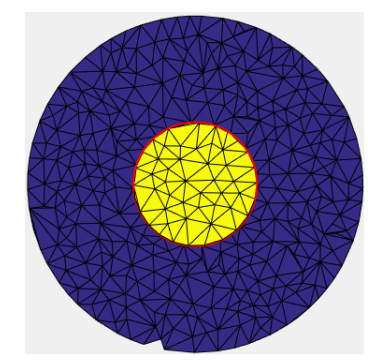

(c) Defect detection in blue

Fig. 15 Displacements (in pixels) and identified regions of defects

\section{Application}

For this application, tests are conducted in the machinetool environment ${ }^{1}$ using a cylindrical part, whose diameter is $150 \mathrm{~mm}$ and its height is $3 \mathrm{~mm}$, machined in

\footnotetext{
1 Mikron UCP710
}

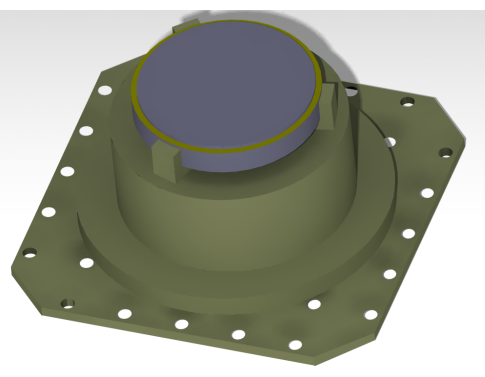

(a) Definition of the nominal part

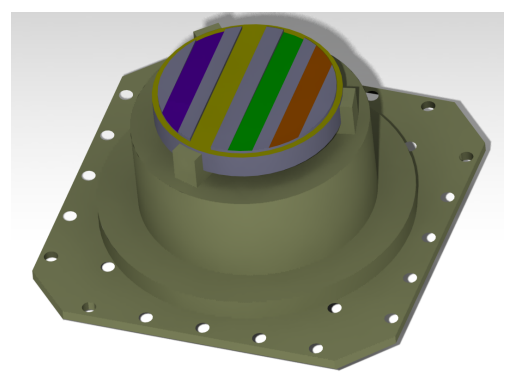

(b) Definition of the machined part

Fig. 16 Description of the studied part

a raw cylinder of diameter $160 \mathrm{~mm}$. The CAD-model of the studied part and its set-up are shown in figure 16(a). Defects are artificially created by machining four grooves (figure 16(b)). The bottom of each groove is defined with different profiles (figure 17). Grooves 1 and 3 are uniform with a constant depth of $0.1 \mathrm{~mm}$ and $3 \mathrm{~mm}$ respectively. Groove 2 and 4 present different slopes. All these grooves are designed in order to test the ability of the stereo-vision system to discriminate various magnitudes of defects. In addition, another more common measuring system is used to detect and measure deviations. This on machine system consists of the Zephyr laser-plane sensor ${ }^{2}$ mounted in the spindle of the machine tool with a dedicated set-up and a standard HSK 63A tool holder attachment (see figure 18). The application of our approach consists of the following steps:

1. Machining of the nominal part

2. Machining of the grooves corresponding to the defects to be measured

3. Measurement of the part using the laser-plane sensor

4. Calibration of the stereo-vision system using the CAD-based appraoch (shooting of 2 pictures)

5. Defect detection using our stereo-DIC approach

2 www.kreon3d.com 


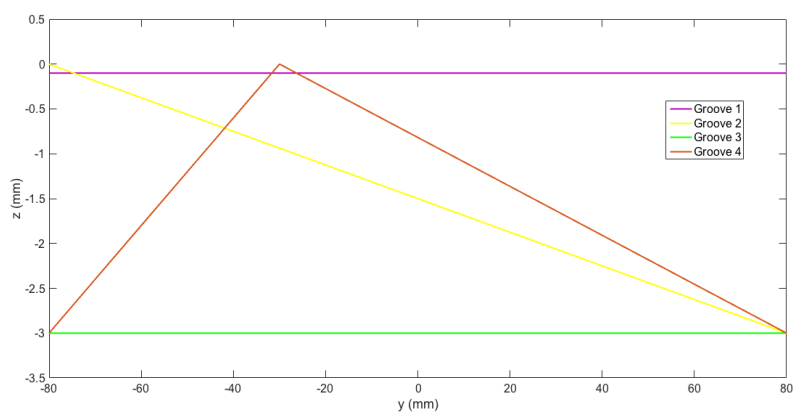

Fig. 17 Description of the grooves

\subsection{Laser-plane sensor measurement}

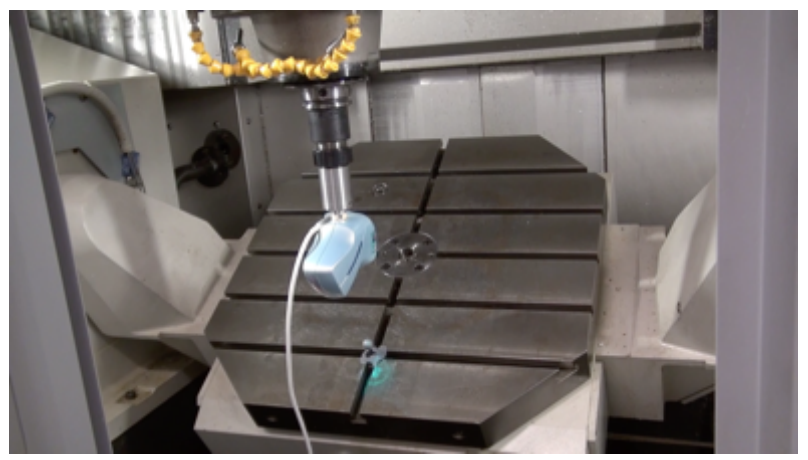

Fig. 18 Description of the in-situ measurement system with Zephyr laser-plane sensor

Only one sensor orientation is used to measure the part surface in order to avoid overlapping errors and to reduce deviations due to orientation changes [1]. Furthermore, the system assessment through the QualiPSo protocol [13] gives a measurement noise of $13 \mu \mathrm{m}$ and a measurement accuracy of $1.3 \mu \mathrm{m} / \mathrm{m}$. Once the surface is measured, the digitized point cloud and the CAD-model are registered based on the Iterative Closest Point algorithm, and deviations are thus calculated. Results are reported in figure 19. Grooves 1 to 4 are displayed from left to right.

Due to its low depth $(0.1 \mathrm{~mm}$ depth $)$, the presence of groove 1 is not quite observable. The depth value of groove 3 is greater than the one expected at $3 \mathrm{~mm}$. This is likely due to registration errors or uncertainties related to the machining process. The slope of groove 2 and the slope change in groove 4 are clearly visible.

\subsection{Stereo-vision measurement and stereo-DIC analysis}

The measurement is performed using two 14.4 Mpixels Cameras. Left and right images are reported in figure

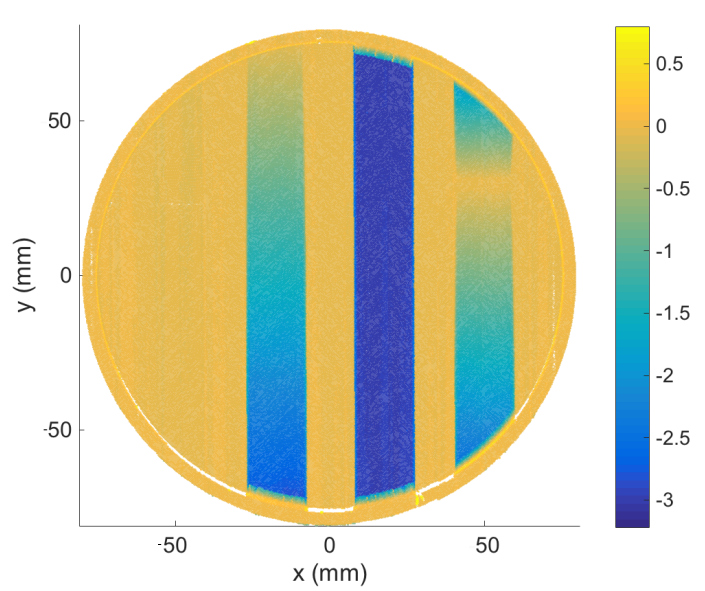

Fig. 19 Measured deviations ( $\mathrm{mm}$ ) with laser-plane sensor

20. To make stereo-DIC analysis possible, a random pattern using black and white painting must be projected onto the part(figure 20(b)). For this purpose, a DLP projector is used, with a $640 \times 320$ pixels resolution, and it is approximately located at one meter of the part. the system calibration is performed (see section 3.1), providing the calibration noises of respectively $\sigma_{0}^{l}=1.70$ pixel for the left camera and $\sigma_{0}^{r}=1.91$ pixel for the right.

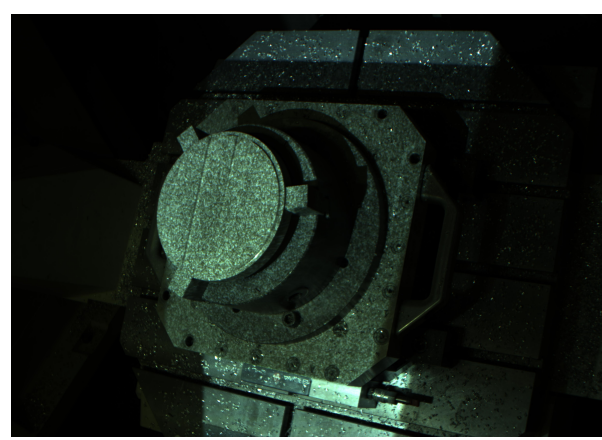

(a) Right picture
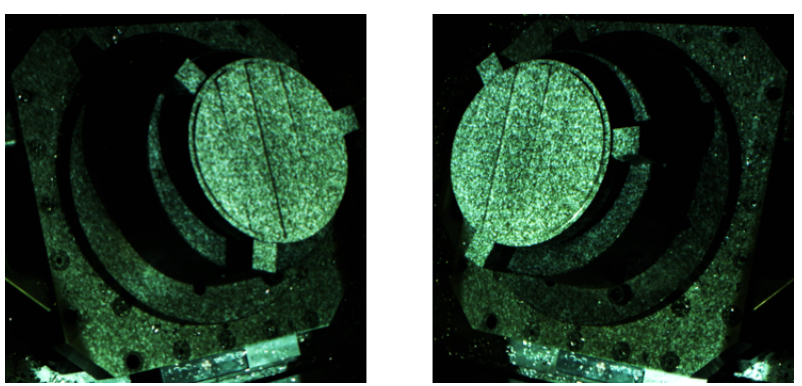

(b) Detailed of the left and right images of the studied part

Fig. 20 Description of the studied part 
Our approach is applied considering a planar nominal part limited by a $140 \mathrm{~mm}$ diameter disk. The average mesh size is about $2 \mathrm{~mm}$. After stereo-DIC application, the estimated displacements are displayed in figures 21(b) and 21(a). Results are quite comparable to those obtained using a laser-plane sensor. In particular, results show that grooves 2 to 4 are identified as defect areas on a large part (figure 21(c)). These results are consistent with the measurements performed using the laser-plan sensor. Nevertheless, the borders of the defect zones are less clearly defined than when using the laser sensor. This is due to the mesh size which should be smaller. For these specific zones, a finer description of the part (refined meshing) should lead to a better identification.

However, area boundaries are poorly identified. This is understandable given that the groove edges are vertical walls, which are not defined in the nominal model. Another explanation comes from visibility. In fact, in a real environment, some portions of the groove edge are only observed by one camera. This observation shows the limitations of the proposed method. Indeed, our method is based on small successive displacements of a nominal shape using the Newton-Raphson algorithm, and to be efficient, the geometry of the measured defect should be close enough from its nominal model.

Groove 1 and the parts of grooves 2 and 4 in the shadow are not detected. This is due to experimental conditions. Indeed, due to the relatively distant position of the cameras, the useful area of each image (figure 20(a)), and therefore the number of pixels, is reduced. This could be corrected by using more adapted lens so that the image only covers the part. Other ways of improvement are possible such as decreasing the mesh size or using a higher resolution projector. Nevertheless, these improvements will increase calculation time but this may be compensated by the determination of the displacement for each facet with GPU computation. Another source of errors comes from the identification of the set-up configuration in the machine-tool frame relatively to the CAD-model frame. These errors lead to residual errors which can be observed in the lower left. This could be simply corrected by carrying out a reference machining on the part. However, our approach is promising as it gives a good idea of the defect location, and can be used during the pre-measurement step.

\section{Conclusion}

Integrating inspection procedures in the machining process contributes to process optimization. However, to be highly efficient, Machining and Inspection Process

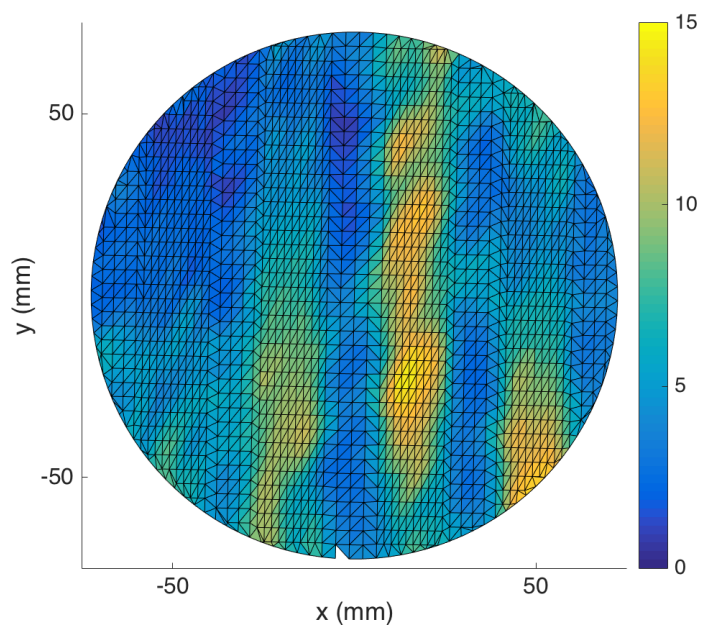

(a) Estimation of the facet displacements $D_{i}^{g}$ (in pixels) in the left camera

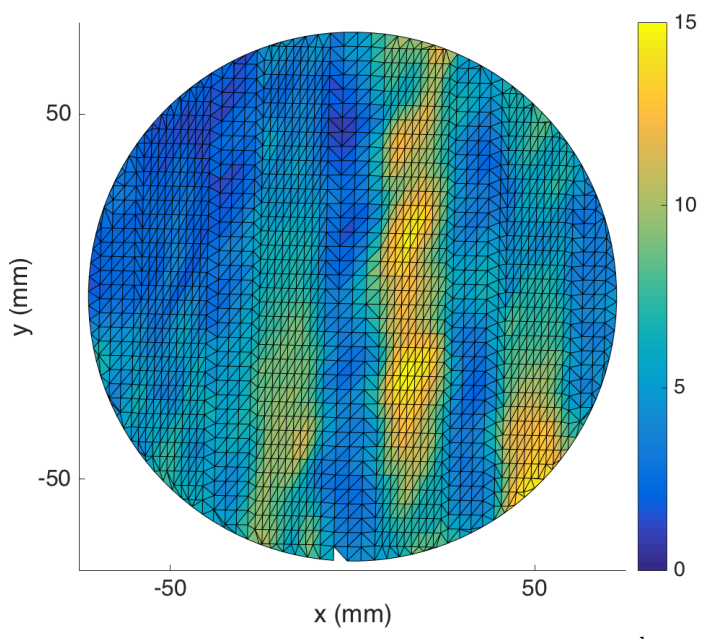

(b) Estimation of the facet displacements $D_{i}^{d}$ (in pixels) in the right camera

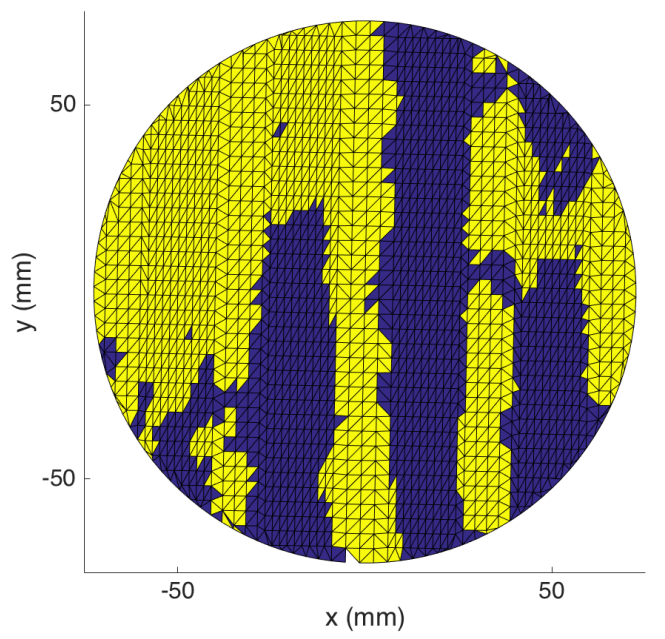

(c) Defect detection in blue

Fig. 21 Displacements(in pixels) and identified regions of defects 
Planning must reach a high level of integration which can be achieved by the use of in-situ measurement. In this paper, we proposed an approach for MIPP based on in-situ measurements. Integrating inspection procedures during the manufacturing process leads to new challenges, such as adapting manufacturing and inspection plans so as not to penalize production costs. The originality of the process planning we propose is to include a step of pre-measurement to localize defects without necessarily quantifying them before the measurement step itself.For the step of pre-measurement, a vision-based system is adopted in this work for its simplicity of implementation within the machine-tool environment, and its rapidity to acquire images. The novel method we develop for defect detection relies on stereoDIC. The originality is the use of a tessellated representation of the CAD model to define regions of interest in the two images. First applications have highlighted the relevance of our approach but have also brought out some key points. First, the calibration noise is a significant parameter that acts as the resolution of our measuring system. Then, measuring conditions must be controlled. In particular, the relative configuration camera/part must be chosen so as shadow areas are limited. Moreover, the resolution of the pattern projected onto the part must be consistent with the order of magnitude of the defect to be identified. Finally, an initial step of frame registration must be performed, probably using the machining of a reference plane, in order to align the actual machine-tool frame with the CAM frame. All these points represent improvements that will be addressed in future work.

\section{References}

1. Bešić, I., Gestel, N.V., Kruth, J.P., Bleys, P., Hodolić, J.: Accuracy improvement of laser line scanning for feature measurements on CMM. Opt. Lasers Eng. 49(11), 12741280 (2011)

2. Bourdet, P., Mathieu, L., Lartigue, C., Ballu, A.: The concept of the small displacement torsor in metrology. Series on advances in Mathematics for Applied Sciences 40, 110-122 (1996)

3. Chen, F., Su, C.: Vision-based automated inspection system in computer integrated manufacturing. The International Journal of Advanced Manufacturing Technology 11(3), 206-213 (1996)

4. Cho, M.W., Lee, H., Yoon, G.S., Choi, J.H.: A computeraided inspection planning system for on-machine measurement part ii: Local inspection planning . KSME International Journal 18(8), 1358-1367 (2004)

5. Cho, M.W., Seo, T.I.: Inspection planning strategy for the on-machine measurement process based on cad/cam/cai integration. The International Journal of Advanced Manufacturing Technology 19(8), 607-617 (2002)
6. Dubreuil, L., Quinsat, Y., Lartigue, C.: Multi-sensor approach for multi-scale machining defect detection. In: Joint Conference on Mechanical, Design Engineering and Advanced Manufacturing, Toulouse, pp. 1-7 (2014)

7. Dubreuil, L., Quinsat, Y., Lartigue, C.: Calibration based on part set-up measurement for on-machine inspection using vision. International Journal on Interactive Design and Manufacturing (IJIDeM) 9(4), 317-323 (2015)

8. Harvent, J.: Shape measurement using a multiple-view Digital Image Correlation (DIC) method: application to the inspection of large-scale aerospace structures. Theses, Université Paul Sabatier - Toulouse III (2010)

9. Harvent, J., Coudrin, B., Brèthes, L., Orteu, J.J., Devy, M.: Shape measurement using a new 3d-dic algorithm that preserves sharp edges. In: SEM 2013 Annual Conference on Experimental and Applied Mechanics, Lombard, Ilinois (USA), 3-6 June 2013. (2013)

10. Lavest, J.M., Viala, M., Dhome, M.: Computer Vision ECCV'98: 5th European Conference on Computer Vision Freiburg, Germany, June, 2-6, 1998 Proceedings, Volume I, chap. Do we really need an accurate calibration pattern to achieve a reliable camera calibration?, pp. 158-174. Springer Berlin Heidelberg (1998)

11. Lee, H., Cho, M.W., Yoon, G.S., Choi, J.H.: A computeraided inspection planning system for on-machine measurement part i: Global inspection planning . KSME International Journal 18(8), 1349-1357 (2004)

12. Mears, L., Roth, J., Djurdjanovic, D., Yang, X., Kurfess, T.: Quality and inspection of machining operations: Cmm integration to the machine tool. ASME. J. Manuf. Sci. Eng 131(5), 1-13 (2009)

13. Mehdi-Souzani, C., Quinsat, Y., Lartigue, C., Bourdet, P.: A knowledge database of qualified digitizing systems for the selection of the best system according to the application. CIRP Journal of Manufacturing Science and Technology 13, 15-23 (2016)

14. Poulhaon, F., Leygue, A., M.Rauch, Hascoët, J.Y., Chinesta, F.: Simulation-based adaptative toolpath generation in milling processes. Int. J. Machining and Machinability of Materials 15(3-4), 263-284 (2014)

15. Quinsat, Y., Tournier, C.: In situ non-contact measurements of surface roughness. Precision Engineering 36(1), $97-103$ (2012)

16. Shahabi, H., Ratnam, M.: Simulation and measurement of surface roughness via grey scale image of tool in finish turning. Precision Engineering 43, 146 - 153 (2016)

17. Sutton, M., Orteu, J.J., Schreier, H.: Image Correlation for Shape, Motion and Deformation Measurements: Basic Concepts, Theory and Applications. Springer Publishing Company, Incorporated (2009)

18. Vacharanukul, K., Mekid, S.: In-process dimensional inspection sensors. Measurement 38(3), $204-218$ (2005)

19. Zhao, F., Xu, X., Xie, S.: Step-nc enabled on-line inspection in support of closed-loop machining. Robotics and Computer-Integrated Manufacturing 24(2), 200 - 216 (2008)

20. Zhao, H., Nabil, A., Pierre, B.: A PLM-Based MultiSensor Integration Measurement System for Geometry Processing, pp. 469-484. John Wiley \& Sons, Inc. (2010) 